16 Illei G, Morgan DML. Polyamine oxidase activity in human pregnancy serum. Br $\mathcal{F}$ Obstet Gynaecol 1979;86:878-81.

17 Illei G, Morgan DML. The distribution of polyamine-oxidase activity in the fetomaternal compartments. Br $\mathcal{F}$ Obstet Gynaecol 1979;86:873-7.

18 Foley GE, Lazarus H, Farber S, Uzman BG, Boone RA, McCarthy RE. Continuous culture cf human lymphoblasts from peripheral blood of a child with acute leukaemia. Cancer 1965;18:522-9.

19 Sly WS, Sekkon GS, Kennett R, Bodmer WF, Bodmer J. Permanent lymphoid lines from genetically marked lymphocytes: success with lymphocytes recovered from frozen storage. Tissue Antigens 1976;7: 165-72.

${ }^{20}$ Morton HJ. Survey of commercially available tissue culture media. In Vitro 1970;6:89-108

${ }^{21}$ Kaplan J, Shope RC, Peterson WD. Epstein-Barr virus-negative human malignant T-cell lines. F Exp Med 1974;139:1070-76.

22 Ross G. Identification of human lymphocyte subpopulations by surface marker analysis. Blood 1979;53:799-811.

${ }^{23}$ Kaplan J, Mastrangelo R, Peterson WD. Childhood lymphoblastic lymphoma, a cancer of thymus-derived lymphocytes. Cancer Res 1974; 34:521-5.

24 Uzman BG, Foley GE, Farber S, Lazarus H. Morphologic variations in human leukaemic lymphoblasts (CCRF-CEM cells) after long-term culture and exposure to chemotherapeutic agents. Cancer 1966;19: 1725-42.

${ }^{25}$ Klopper A, Hughes G. Placental secretion of oestrogens and protein hormones. Arch Gynaekol 1978;225:171-7.

${ }^{26}$ O'Brien J, Knight S, Quick NA, Moore EH; Platt AS. A simple technique for harvesting lymphocytes cultured in Terasaki plates. $\mathcal{f}$ Immunol Methods 1979;27:219-23.
27 Morgan DML, Ferluga J, Allison AC. Polyamine oxidase and macrophage function. In: Gaugas JM, ed. Polyamines in biomedical research. Chichester: John Wiley (in press)

${ }^{28}$ Morgan DML, Illei G. Radiochemical estimation of polyamine oxidase in serum and tissue in pregnancy. Biochem Med (in press).

${ }^{29}$ Gaugas JM, Dewey DL. Evidence for serum binding of oxidised spermine and its potent $G_{1}$-phase arrest of cell proliferation. Br $\mathcal{F}$ Cancer 1979; $39: 548-57$.

${ }^{30}$ Rennert OM, Lawson DL, Skukla JB, Miale TD. Cerebrospinal fluid polyamine monitoring in central nervous system leukaemia. Clin Chim Acta $1977 ; 75: 365-9$.

31 Rennert OM, Miale T, Shukla J, Frias J. Polyamine concentrations in bone marrow aspirates of children with leukaemia and other malignancies. Blood 1976;47:695-701.

32 Gunga CK, Sheth AR, Gunaga KP, Rao SS, Purandane MC. Polyamines in human placenta. Indian $\mathcal{F}$ Biochem Biophys 1972;9:272-4.

33 Ponta R, Servillo L, Abbruzzese A, Pietra GD. Automated chromatographic analysis of human placenta polyamines. Biochem Med 1978;19: 143-7.

${ }^{34}$ Southren AL, Kobayaski Y, Weingold AB, et al. Serial plasma d: nine oxidase (DAO) assays in first and second trimester complicati $\mathrm{s}$ of pregnancy. Am 7 Obstet Gynecol 1966;96:502-10.

35 Southren AL, Kobayaski Y, Carmody Y, et al. Serial measurem is of plasma diamine oxidase (DAO) during normal human pregnancy .ry an improved method. Evidence for the presence of a circulatin!. DAO inhibitor. Am 7 Obstet Gynecol 1966;95:615-20.

(Accepted 5 February 1980)

\title{
Idiopathic carpal tunnel syndrome caused by carpal stenosis
}

\author{
S DEKEL，T PAPAIOANNOU，G RUSHWORTH， R COATES
}

\section{Summary and conclusions}

Computed tomography was used to measure the crosssectional area of the carpal canals in normal controls of both sexes and in women with idiopathic carpal tunnel syndrome. The women controls had significantly smaller carpal canals than the men controls both proximally and distally. In the patients both the proximal and distal cross-sectional areas were significantly reduced compared with the women controls. The measurements showed that carpal canal stenosis is associated with idiopathic carpal tunnel syndrome, narrowing of the canal is bilateral in patients who have unilateral symptoms, and narrowing is greater in the proximal carpal canal. There was no correlation between age and the size of the canal.

The difference in the size of the carpal canal between normal men and women might explain the tendency of women to develop carpal tunnel syndrome. The lack of correlation between age and the size of the canal suggests that stenosis of the carpal canal is inherited rather than acquired. Symptoms arise only later in life, when degenerative changes in the content or the walls of the carpal canal compete with the median nerve for space and its function becomes impaired by compression.

Nuffield Department of Orthopaedic Surgery, Nuffield Orthopaedic Centre, and University Department of Clinical Neurology, Churchill Hospital, Oxford

$S$ DEKEL, MD, clinical lecturer in orthopaedic surgery

T PAPAIOANNOU, MD, research fellow

G RUSHWORTH, DM, university lecturer in clinical neurophysiology

$R$ COATES, FRCS, clinical lecturer in orthopaedic surgery

\section{Introduction}

The carpal tunnel syndrome is the most common peripheral nerve lesion and may be produced by several different conditions. In general, any pathological process that reduces the crosssectional area of the carpal canal or swells its contents will compress the median nerve and produce the clinical manifestations of the syndrome. The carpal tunnel syndrome is well documented after bone fractures near the wrist, ${ }^{1}$ dislocation of the carpal bones, ${ }^{2}$ and soft-tissue tumours. ${ }^{3}$ In about half of the patients with the condition, however, the cause cannot be determined. Such patients with idiopathic carpal tunnel syndrome are often women approaching the menopause. ${ }^{4}$ It has been suggested $^{5}$ that the idiopathic carpal tunnel syndrome is the result of anatomical narrowing of the carpal canal in women combined with oedema.

The aim of our study was to use computed tomography to measure the transverse dimensions of the carpal canal in patients with idiopathic carpal tunnel syndrome and in normal men and women, and to compare the results in the three groups.

\section{Methods}

The normal subjects comprised 19 women (mean age 39) and 14 men (mean age 42). We also studied 26 women with idiopathic carpal tunnel syndrome, in whom the diagnosis was based on the history and results of clinical examination and of electrophysiological studies on the median, ulnar, and radial nerves of the hands (details of these measurements will be reported separately).

Altogether we studied 42 hands: 16 patients had the syndrome in both hands and 10 in only one. Patients who might have one of the recognised diseases associated with carpal tunnel syndrome were excluded. The following complaints were associated with the condition: nocturnal pain (40 hands), paraesthesiae (40), pain in the hand (24), clumsiness (23), and pain in the forearm (21). The syndrome was 
related to pregnancy (14 hands) and to the patient's period (seven) Findings were as follows: positive reaction to Phalen's test 31 hands; hypoaesthesia 26; distal tingling on percussion (Tinel's sign) 22 ; weakness of abductor pollicis brevis 18; wasting of abductor pollicis brevis 18; swelling proximal to carpal canal 15; positive response to tourniquet test 14 (not recorded in 14 hands); and diminished sweating nine.

Both hands of patients and controls were radiographed simultaneously and a special moulded thermoplastic splint used to secure a standard position of the wrist during the computed tomography (CT 1010 F35). Several radiographs were taken through both carpal canals, and pairs of transverse sections of only the proximal (fig 1) and distal (fig 2) parts of the canals were then mounted in $5 \times 5 \mathrm{~cm}$ slide-binders and projected on to a table digitiser connected to a computer (Digital Equipment Corporation PDP 31/34). As the flexor

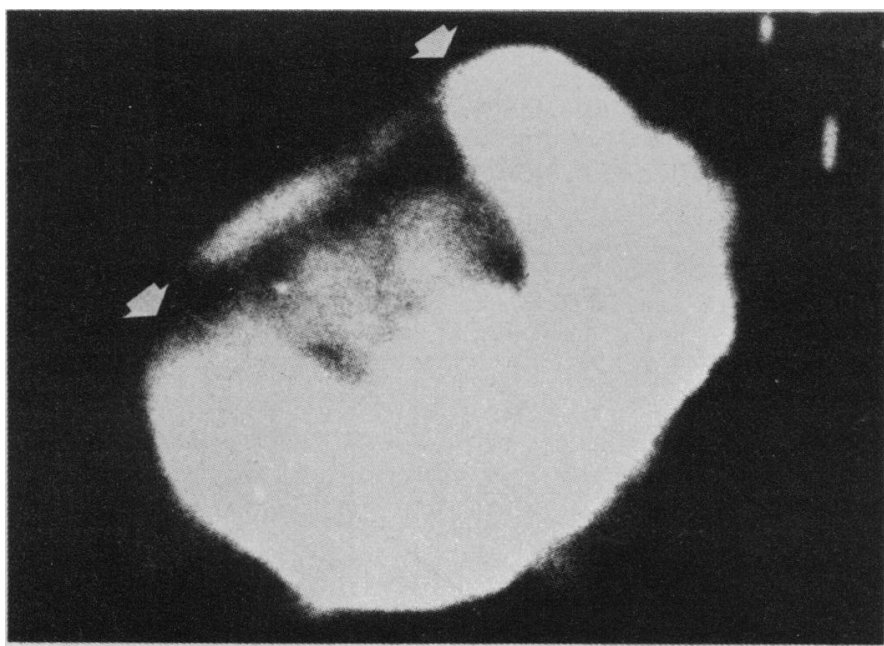

FIG 1-Tomogram of proximal area of carpal tunnel in female control. Note pisiform bone on right and tubercle of scaphoid on left (arrows).

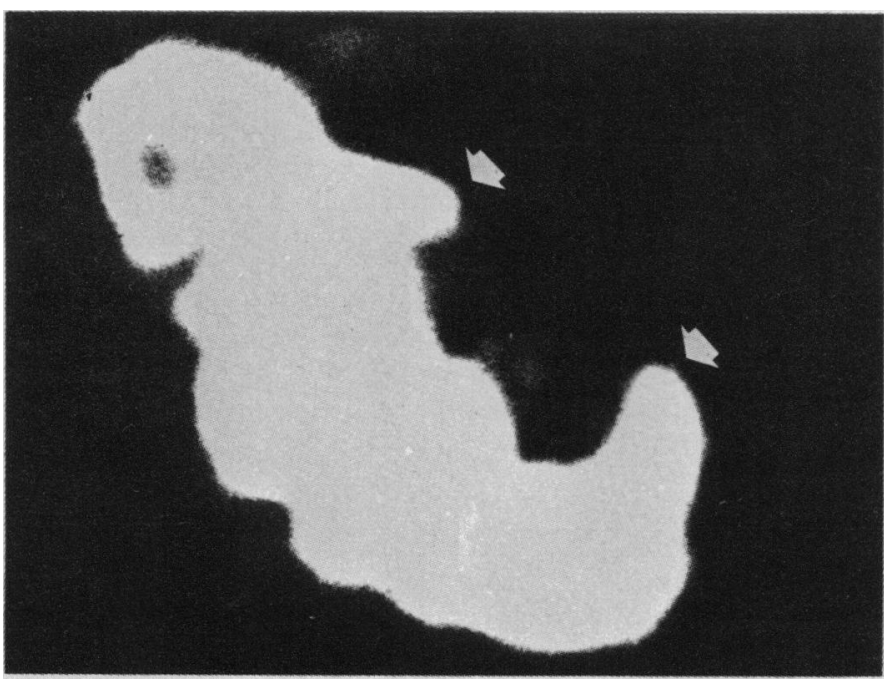

FIG 2-Tomogram of distal area of carpal tunnel in male control. Note hook of hamate on right and tubercle of trapezium on left (arrows).

retinaculum was not clearly visible on the radiographs a line was drawn from the scaphoid tubercle to the pisiform bone and from the trapezium to the hook of the hamate corresponding to the anatomical attachment of this ligament. ${ }^{6}$ The area enclosed by the carpal bones and this line was outlined on the digitiser and fed into the computer, which then gave a direct measurement of the area. The measurements were corrected to the actual area of the carpal tunnel by multiplying by a correction factor determined from an identically processed radiograph of a rectangular metal box of known cross-sectional area. The measured area of the carpal canal is slightly less than the true area, as the shape of the flexor retinaculum is a gentle curve and not a straight line from its attachments (fig 3). When two radiographs passed through the same anatomical area they were both measured and the mean used. The standard Student's $t$ test and paired $t$ test were used for statistical analysis of the data.

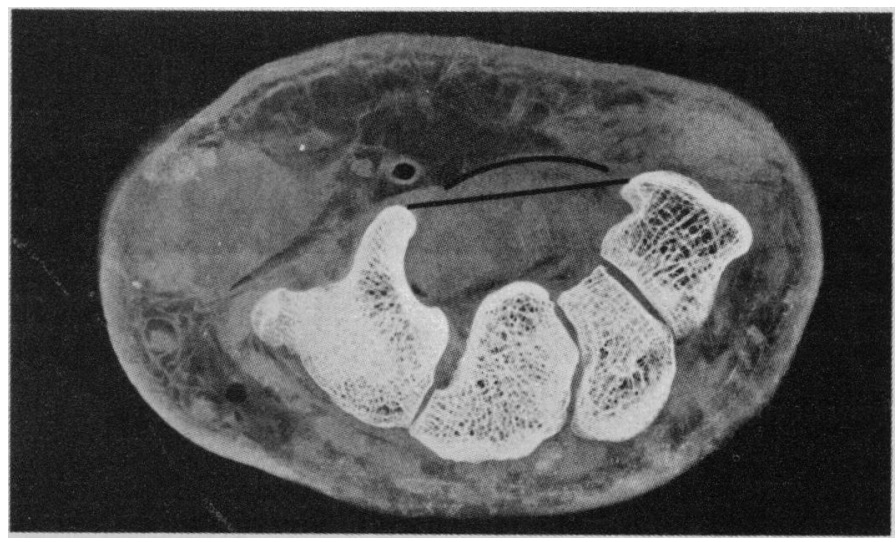

FIG 3-Cross-section through distal part of carpal canal of fresh pathological specimen. Note that the carpal ligament, attached to the hook of the hamate and trapezium, is. gently curved. As the carpal canal was not clearly defined in the radiographs a line was drawn between the bones and the area enclosed by this line and the carpal bones measured.

\section{Results}

The cross-sectional area of the proximal part of the carpal canal was significantly smaller $(p<0.001)$ in the female controls than in the male controls (mean \pm SEM area $213.7 \pm 4.8 \mathrm{~mm}^{2} v 279.9 \pm 8.3 \mathrm{~mm}^{2}$; fig 4), and the area in the patients $\left(184 \cdot 1 \pm 4.0 \mathrm{~mm}^{2}\right)$ was significantly smaller $(p<0.001)$ than that in the female controls.

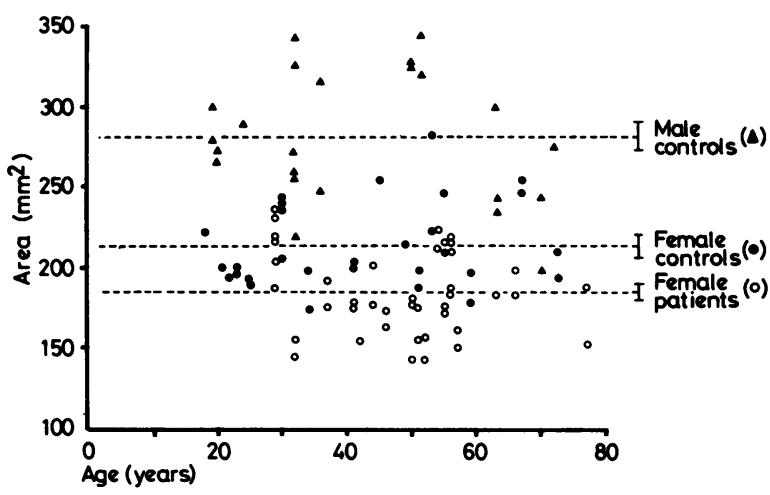

FIG 4-Proximal cross-sectional areas of carpal canal in female patients (41 hands), female controls ( 29 hands), and male controls (23 hands). Dotted lines indicate means, and bars SEMs, in each group.

Measurements of the distal part of the canal showed similar differences. This area was significantly smaller $(p<0.001)$ in the female controls than in the male controls $\left(209.3 \pm 7.7 \mathrm{~mm}^{2} v 254.1 \pm 7.8 \mathrm{~mm}^{2}\right.$; fig 5), and the area in the patients $\left(188.0 \pm 6.5 \mathrm{~mm}^{2}\right)$ was significantly smaller $(p<0.05)$ than that in the female controls.

In all three groups the mean cross-sectional area of the carpal canal of the dominant hand was greater than that of the non-dominant hand, but the differences were not significant (paired $t$ test). In the two control groups the mean proximal area was greater than the mean distal area, but the differences were significant only in the men $(p<0.05)$. In the patients, however, the reverse was found, with the mean proximal area being smaller than the distal area, but this difference was not significant.

No correlation was found between either the proximal or the distal areas of the carpal canal and the age of the subject in any of the three groups. In four out of five patients with unilateral symptoms in whom radiographs of the proximal parts of both canals were obtained a positive correlation was found between the area of the canal and the 
side of the symptoms. This difference, however, was not significant $\left(167.7 \pm 6.9 \mathrm{~mm}^{2} v 173.6 \pm 10.8 \mathrm{~mm}^{2}\right.$, paired $t$ test $\left.\mathrm{p}>0.05\right)$. In five patients with bilateral symptoms that were worse in one hand, however, no positive correlation was found between the proximal area and the hand that was more severely affected.

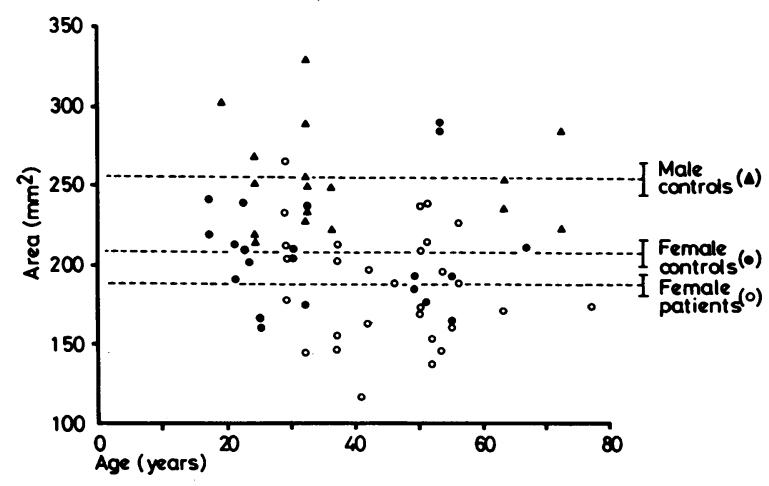

FIG 5-Distal cross-sectional areas of carpal canal in female patients ( 29 hands), female controls ( 21 hands), and male controls (17 hands). Dotted lines indicate means, and bars SEMs, in each group.

\section{Discussion}

The pathological changes producing the idiopathic carpal tunnel syndrome have long been controversial. Watson-Jones? suggested that the condition resulted from thickening of the flexor retinaculum, which compressed the median nerve. Phalen and Kendrick ${ }^{8}$ showed that the most common factors leading to the carpal tunnel syndrome were flexor tenosynovitis and fibrosis, which they suggested were caused by localised rheumatoid disease. Tanzer ${ }^{910}$ measured the thickness of the flexor retinaculum during operative decompression of the carpal tunnel in patients with the carpal tunnel syndrome and compared his measurements with those found at routine necropsy in the same number of patients with presumed normal hands. $\mathrm{He}$ found no difference between the two groups; nor was there any difference in the synovium. Structural narrowing of the carpal canal combined with an increase in the volume of its content was suggested ${ }^{5}$ as the cause of the idiopathic carpal tunnel syndrome, but no evidence was forthcoming.

Our results show that an association exists between stenosis of the carpal canal and the idiopathic carpal tunnel syndrome. Neurological symptoms appear later in life when degenerative changes in the content or the walls of the carpal canal produce compression of the median nerve. Symptoms may appear for the first time during pregnancy owing to fluid retention and compression of the median nerve by swelling of the contents of the canal.

No clear correlation existed between age and the crosssectional area of the carpal canal in any of the three groups studied (figs 4 and 5), which suggests that carpal stenosis is genetically determined rather than acquired. This would explain the strong familial incidence of the condition. ${ }^{4}$ The narrowing, however, affects the proximal rather than the distal part of the canal, and this would correlate with the clinical observations ${ }^{48}$ that at operation the maximum compression of the median nerve is in this area. Careful decompression of the proximal region of the carpal canal is therefore important for operative success. The difference in the distal area of the carpal canal between the female patients and controls was only marginally significant $(p<0.05)$, but in some patients this area was more than one standard deviation below the mean control value. This suggests that the idiopathic carpal tunnel syndrome might be the result of either proximal or distal stenosis, or both. The observation that stenosis occurred in the proximal part of the carpal canal in the non-symptomatic hand of patients with carpal tunnel syndrome might explain the high proportion of patients who eventually have the syndrome in both hands. If stenosis is always bilateral a prophylactic operation on the nonsymptomatic hand might prevent subsequent damage to the median nerve.

Finally, our results show that both the proximal and distal cross-sectional areas of the carpal canal in women are smaller by $25 \%$ than the corresponding areas in men, which might explain the high incidence of the idiopathic carpal tunnel syndrome in women.

We are grateful to Ann Colwyn and Sarah Hunt for technical help; Sylvia Bird and Jacqueline Cole for help with the neurophysiologica examinations; Margaret Proudlock-Dunbar for typing the manuscript; and Graham Bartlett for the illustrations. We are also grateful to Professor R B Duthie for his constant encouragement.

\section{References}

1 Zachary RB. Thenar palsy due to compression of the median nerve in the carpal tunnel. Surg Gynecol Obstet 1945;81:213-7.

2 Watson-Jones R. Primary nerve lesions in injuries of the elbow and wrist. f Bone foint Surg 1930;12:121-40.

3 Sunderland S. Nerves and nerve injuries. Edinburgh: Churchill Livingstone, 1978:711-27.

4 Phalen GS. The carpal tunnel syndrome. Clin Orthop $1972 ; 83: 29-40$.

- Bradley WG. Disorders of the peripheral nerves. Oxford: Blackwell, 1974:152-5.

- Kaplan EB. Functional and surgical anatomy of the hand. Philadelphia: Lippincott, 1965:187-8.

7 Watson-Jones R. Leri's pleonosteosis, carpal tunnel compression of the medial nerves and Morton's metatarsalgia. F Bone foint Surg 1949, 31B:560-71.

8 Phalen GS, Kendrick JI. Compression neuropathy of the median nerve in the carpal tunnel. $\mathcal{F} A M A 1957 ; 164: 524-30$.

- Tanzer RC. The carpal tunnel syndrome: a clinical and anatomical study. f Bone foint Surg 1959;41A:626-34.

10 Tanzer RC. The carpal tunnel syndrome. Clin Orthop 1959;15:171-9.

(Accepted 1 February 1980)

ONE HUNDRED YEARS AGO The English railway-companies, in denying foot-warmers to their travellers in cold weather, are more than parsimonious-they inflict a severe punishment and, it may well be, from time to time, a dangerous injury on their travellers. It is very well known to physicians that there are many persons, even in sound health, to whom coldness of the feet is much more than a discomfort. It is the signal for internal congestions, quite out of proportion to the mere lowering of temperature of the part, but due to reflex impressions on internal organs. We are cognisant of more than one case in which coldness of the feet means always an attack of congestion of the kidneys. The persons who are particularly liable to injury from this cause are, especially, literary men and others of sedentary pursuits; but, besides these, there are a numerous class of invalids to whom the want of foot-warmers in cold weather is a source of pain and injury. England is the only civilised country with which we are acquainted, in which adequate provision is not made for supplying this necessity of railway travelling in cold weather. Everyone knows that in France, and, indeed, throughout the continent, as in America, either the carriages are heated or the supply of foot-warmers is abundant, regular, and gratuitous. In England, they are often not to be had, even by paying for them, while other than firstclass passengers are absolutely denied this very necessary comfort. It may be hoped that public attention being directed to the subject will induce the directors to give the necessary consideration to what would, no doubt, in the first instance, involve a somewhat costly addition to their permanent stock, and some increase of labour. To continue, however, to deny such a simple and elementary necessity of travelling in cold weather, is hardly justifiable. It is more than probable that the hardships of travelling without it prevent many from taking journeys in severe weather if they can avoid it; and that it would not be, even from a money point of view, bad policy for railway-directors to provide railway-travellers with the means of keeping the feet warm in cold weather. (British Medical fournal, 1880.) 